\title{
A nanoscale investigation of mechanical, thermal stability and electrical conductivity properties of reinforced thermoplastic polyurethane/graphene nanocomposite
}

\author{
Irene S. Fahim ${ }^{1}$, Wael Mamdouh ${ }^{2,3, ~}$, Hanadi A. G. Salem ${ }^{1,3}$ \\ ${ }^{1}$ Department of Mechanical Engineering, School of Sciences and Engineering, The American University in Cairo, AUC Avenue, New \\ Cairo 11835, Egypt \\ ${ }^{2}$ Department of Chemistry, School of Sciences and Engineering, The American University in Cairo, AUC Avenue, P.O. Box 74, New Cairo \\ 11835, Egypt \\ ${ }^{3}$ Yousef Jameel Science and Technology Research center, School of Sciences and Engineering, The American University in Cairo, AUC \\ Avenue, New Cairo 11835, Egypt
}

\section{Email address:}

rico4000@aucegypt.edu(I. S. Fahim), wael_mamdouh@aucegypt.edu(W. Mamdouh), madkour@aucegypt.edu(T. M. Madkour), hgsalem@aucegypt.edu(H. A. G. Salem)

\section{To cite this article:}

Irene S. Fahim, Wael Mamdouh, Hanadi A. G.Salem. A Nanoscale Investigation of Mechanical, Thermal Stability and Electrical Conductivity Properties of Reinforced Thermoplastic Polyurethane/Graphene Nanocomposite. American Journal of Nanoscience and Nanotechnology. Vol. 1, No. 1, 2013, pp. 31-40. doi: 10.11648/j.nano.20130101.17

\begin{abstract}
In the current research work reinforcement of a thermoplastic polyurethane (TPU) polymer with graphene powder ( $\mathrm{G}$, in the form of particles) as a nanofiller material by in-situ and ex-situ mixing of various weight fractions of $\mathrm{G}$ is reported. In addition, investigation on the effect of the weight fraction of $\mathrm{G}$ on the mechanical properties of the resulting TPU/graphene (TPU/G) nanocomposites is conducted. A number of different preparation methods have been employed in order to investigate the influence of the preparation process on the resulting TPU/G nanocomposites. Solvent (wet) mixing and mechanical (dry) mixing processes have been used. Significant enhancement in compressive strength, melt flow index and electrical conductivity were observed by employing the different mixing processes when compared with the pure TPU polymer processed under similar conditions. However, dry mixing process has shown enhanced mechanical, viscosity and electrical properties compared to wet mixing process. Moreover, dry mixing process has led to the formation of TPU/G nanocomposites with the highest compressive strength at $0.1 \mathrm{wt} \% \mathrm{G}$ compared with 0.5 , and $1 \mathrm{wt} \% \mathrm{G}$. It is believed that the processing technique plays a vital role in producing the desired TPU/G nanocomposites and is also affected by the dispersion of graphene nanofiller particles within the TPU polymer matrix. These results may lead to the development of novel applications of TPU/G nanocomposites across different disciplines.
\end{abstract}

Keywords: Polymer Nanocomposites, Graphene, TPU, Conductivity

\section{Introduction}

Reinforcement of polymeric materials has attracted a lot of attention by which an intensive amount of research effort on modification of polymers by adding inorganic fillers as a second phase has become ubiquitous in polymeric systems. Interestingly, it has been shown that the addition of filler materials (e.g. inorganic particle) of nanoscale dimensions into a polymer matrix is an effective way to develop novel polymer materials with notable properties to meet new industry requirements and to replace some of the existing materials with property limitations. These new reinforced polymer conjugate materials are known as "Polymer Nanocomposites (PNCs)" exhibit unique properties such as stiffness, toughness, heat distortion, temperature and mold shrinkage and show a significant enhancement in thermal, electrical, mechanical, optical, flammability and gas-barrier properties according to their applications. These new PNCs always combine the advantages of corrosion resistance, light weight, and ease of processing from polymers and other functional performance such as electrical conductivity from fillers.[1].

A wide range of fillers has been used such as: calcium carbonate, clay, titanium dioxide, silica, alumina tri-hydrate, carbon or glass fibers, carbon nanotubes, and carbon black 
which serve many different applications such as household goods, packaging, sporting goods, aerospace components, and automobiles[2]. Compared to other types of reinforcement, where $20 \%$ to $40 \%$ filler is needed, nano-fillers offer the possibility of achieving the desired effect with very low filler contents, often in the range of a few weight percent. Nanoparticles are added to metallic, ceramic and polymeric materials as a reinforcement to enhance the mechanical, thermal and physical properties of the parent compound. Owing to the very small filler dimensions, the interface between the matrix and filler is large, even at low filler concentration, and filler properties can be largely transferred to the matrix[3].

Filler particles in the nanometer range have a large surface area-to-volume ratio leading to improved physical properties and chemical reactivity[4]. Among these promising fillers, graphene molecules have shown tremendous applications across a wide spectrum of disciplines ranging from biomolecular nanocomposites and up to molecular electronics. This is mainly due to its exceptional electron transport, mechanical properties, and high surface area[5-6].

Graphene nanoparticles are used as reinforcement for synthetic polymers. Graphene is an isolated single-atom plane of graphite. In 1960, higher basal-plane conductivity of graphite intercalation compounds was discovered compared to that of the original graphite. This was considered a cheaper substitute for metal conductors. The research about graphene has drawn great attention from scientific community since its discovery in 2004. It is considered a promising nanofiller due to its excellent mechanical, thermal and electrical property, combining with its ultra high surface area and economical sources. It is an atomically thick, two-dimensional sheet composed of $s p^{2}$ carbon atoms covalently bonded in a hexagonal manner. There are several structures of graphene including, 0-D fullerenes (buckyballs) that are made by wrapping a section of graphene sheet. 1-D carbon allotropes, carbon nanotubes (CNT) are made by rolling and slicing graphene sheets respectively[7]. Graphene has an affinity to organic compounds and polymers due to the presence of multi-pores, functional acids and $\mathrm{OH}$ groups. This enables polymers to be absorbed into the pores besides its high levels of stiffness, strength, and thermal conductivity[8-9].

Thermoplastic polyurethane (TPU) is a synthetic polymer with outstanding properties. It is a linear segmented block copolymer having hard segments (HS) and soft segments (SS). The SS consist of the long flexible polyether or polyester chains, which interconnect two hard segments. The hard segments act as multifunctional tie points functioning both as physical cross links and reinforcing fillers, while the soft segments form an elastomeric matrix, which accounts for the elastic properties of this polymer. The presence of two soft segments can show different extents of phase separation, offering new possibilities on tuning polymeric matrix properties. Thus, TPU has been used for a variety of applications such as fibers, coatings, adhesives. However, despite good extensibility, abrasion resistance, good solvent resistance and high-temperature performance still low stiffness and tensile strength can be drawbacks in structural applications especially for TPU with low hard segment content. Water vapor and other gas permeability can be another drawback. These weak mechanical and gas barrier properties can be complemented by reinforcing with rigid layered nano-fillers[10-11]. The addition of Graphene to TPU will enhance the properties of TPU polymer to be used in several applications including automobiles, aerospace, injection molded products, coatings, adhesives, fire-retardants, packaging materials, optical integrated circuits, drug delivery, sensors, water filtration systems, dental, medical devices and tissue engineering applications[8].

There are several methods of adding the fillers into the polymeric matrix including solvent mixing, in situ polymerization and direct melt-mixing. Solvent mixing occurs when the solid polymer is dissolved in a selected solvent, to which the filler is added and dispersed by sonication. This is followed by solvent evaporation and the re-solidification of the polymer now containing the dispersed filler. However, there is a concern that the solvents may influence the mechanical performance of the composite and that the sonication energy tends to break the filler into shorter lengths. Moreover the evaporation of large amounts of the solvent will not enhance the mass production[12]. Melt mixing on the other hand by using conventional polymer processing techniques such as extrusion and compression molding are used frequently due to the its high speed and simplicity[13]. After mixing the polymer matrix and the filler, there is an interfacial region between the matrix and filler that is formed. The interfacial region communicates between the polymer matrix and the filler and acquires different properties from the bulk matrix because of its proximity to the surface of the filler[14].

Previous work has been carried out to fabricate several nanocomposites with improved mechanical properties. Reinforcing Aluminum with carbon nanotubes showed a 78\% increase in hardness with $5 \mathrm{wt} \%$ CNTs compared to aluminum without reinforcement[15]. In addition, in a polymer-derived ceramic matrix; silicon-carbon nitride reinforced with CNT showed an improvement from conductivity amounted to $7.6 \times 10^{-2} \mathrm{~S} / \mathrm{cm}$ to 1.71 at $5 \mathrm{wt} \% \mathrm{CNT}[16]$. Moreover, previous works have been carried out with the purpose of characterizing similar nanocomposites with TPU and different fillers. Quan et al. investigated the fabrication of Graphite nanoplatelets (GNP) filled with thermoplastic polyurethane (TPU) polymers using melt blend mixing. The inclusion of GNPs improved the stiffness of TPU and thermal stability of the resulted nanocomposite. It also maintained a long elongation at break value (over 600\%). However, the mixing technique used before melt mixing was solvent mixing[17]. Barik et al. prepared Carbon nanofibres (CNF) based TPU nanocomposites by melt blending, to explore the effect of dispersion and wt. \% loading of CNF on material properties. The thermal stability of the TPU evaluated by Thermo gravimetric analysis (TGA) showed 
significant increase with increased CNF content. It has been observed that storage modulus and glass transition temperature of the TPU matrix increased by the incorporation of CNF. The melting point of soft segments observed from Differential Scanning Calorimetry (DSC) was found to shift towards higher temperature with the inclusion of CNF[18].

\section{Experimental Procedure}

\subsection{Materials}

Graphene is added as a filler to thermoplastic polyurethane (TPU) to produce TPU/G nanocomposites and was provided by Skyspring Nanomaterials, Inc. (USA). G particle size is $(6-8 \mathrm{~nm})$. TPU was prepared as reported previously in the form of pellets[19]. Tetrahydrofuran (THF) (Sigma Aldrich, 99\% purity) was used to dissolve TPU and G. Methanol (Sigma Aldrich, $99.9 \%$ purity) and ethanol (Sigma-Aldrich, $99.5 \%$ purity), were used for cleaning the glassware. The mold material is plated steel with a stainless steel frame. The upper and lower punches are made of polished steel.

\subsection{Mixing Techniques}

The amount of $\mathrm{G}$ added to the TPU was varied so as to obtain TPU/G nanocomposite samples of $0.1,0.5$, and $1 \mathrm{wt} \%$ of $\mathrm{G}$ dispersed in TPU matrix. Two processing techniques were used for the in-situ and ex-situ mixing of graphene and TPU; solvent (wet) mixing and mechanical (dry) mixing.

\subsubsection{Solvent (Wet) Mixing}

TPU pellets were dissolved in THF with constant stirring to form a clear homogeneous solution. $G$ was then added to the TPU solution with continuing stirring for $60 \mathrm{~min}$. The resulting TPU/G nanocomposites were left to dry in air at room temperature.

\subsubsection{Mechanical (Dry) Mixing}

A turbula mixer (Turbula T2F, Switzerland) was used to mix the TPU pellets with G powder in-situ. During the process, the mixing speed was set to $96 \mathrm{rpm}$ and the duration was 1 hour. The mixer works by moving the mixing container in a three dimensional motion of rotation, translation, and inversion

\subsection{Hot Compaction}

Melt processing indicates the blending of filler materials with the melted polymer matrix by the application of shear forces[13]. Two of the most commonly used techniques of melt processing are; i) extrusion and ii) compression molding[20]. The method used in this work is melt processing by compression molding. TPU/G nanocomposites (with 0.1 wt $\%, 0.5 \mathrm{wt} \%$, and $1 \mathrm{wt} \% \mathrm{G}$ weight fraction percentages) were fabricated through a cylindrical mold made of stainless steel to form TPU/G round compact discs. The TPU/G mixture (in the dry form) were placed in a cylindrical mold and then placed on a mechanical press (Carver Laboratory
Press, USA). The mixture is pressed under a pressure of 100 bars; a pressure that is sufficient to prevent leaking of the mixture out of the mold (i.e. flash). Then, the mixture softens and flows into the desired shape (i.e. round compact disc) as the mold temperature reaches $170{ }^{\circ} \mathrm{C}$. The mold is then cooled down and the molding (TPU/G round compact disc) is extracted. During compaction, silicon based grease was used to avoid the sample from sticking to the mold. After the compression molding process, the TPU/G round compact disc was washed thoroughly with distilled water followed by another washing round with ethanol to minimize contamination.

\subsection{Mechanical Testing}

The compressive behavior of TPU/G nanocomposite was evaluated through an axial compression test. The test was conducted using an Instron universal testing machine (Model 3300, Instron, England). For every single TPU/G round compact disc preparation, 7 grams of TPU/G sample was placed on the top of a cylindrical plate, and then another cylindrical plate applies a vertical compression on the sample with a strain rate of $1.3 \mathrm{~mm} / \mathrm{min}$ and the preload was set to $0.1 \mathrm{~N}$. For final adjustment of the specimen (i.e. TPU/G round compact discs) dimensions to be in accordance with ASTM- D 1621 standard, mechanical modification was used to yield height-to-diameter (HD) ratio of $1: 2$. The final TPU/G specimen dimensions were adjusted to be $\mathrm{H}=12.5$ $\mathrm{mm}$ and $\mathrm{D}=25 \mathrm{~mm}$.

\subsection{Scanning Electron Microscopy (SEM)}

The microstructure of the TPU/G compact discs, the homogeneity of the nanocomposite and the dispersion of graphene in The PU matrix were analyzed using a scanning electron microscope (FESEM, Leo Supra 55 - Zeiss Inc., Germany).

\subsection{Image J Analysis}

Image $\mathrm{J}$ was used to display, edit, analyze, and process the SEM images in order to determine the particle size and distribution by creating a density histogram. It is commercially available software used for image processing and analysis. The measurement scale is set from the scale bars of each of the SEM images. A careful selection of the desired particles and features appearing in the SEM images have been taken into account and inserted in the software.

\subsection{Melt Flow Index}

The viscosity of the TPU/G was measured using a melt flow indexer (MFI) (A Ray-Ran Co. England melt flow systems). In this technique, a stainless steel cylinder is placed on top of the TPU/G nanocomposite powders placed inside a column. The selection of the cylinder's weight is in accordance with ASTM D-1238 and the weight is selected to be $2.16 \mathrm{~kg}$ at $210^{\circ} \mathrm{C}$. The procedure is to heat the sample placed inside the column until reaching its melting point at which the cylinder will start flowing through the melt to 
produce stripes of the material. The weight of the resulting material strips was measured every $0.5 \mathrm{~min}$. The specified flow range to drive the melt through the column was in $\mathrm{g} / 10$ $\min$.

\subsection{Thermo Gravimetric Analysis}

Thermo gravimetric analysis (TGA) experiments were carried out to determine the variation in the thermal degradation temperature of polyurethanes by TGA analyzer Q series (Thermo Fisher Scientific, USA). Heating rate of $20{ }^{\circ} \mathrm{C} / \mathrm{min}$ was used to raise the temperature of $10-14 \mathrm{mg}$ samples from room temperature up to $700{ }^{\circ} \mathrm{C}$ under nitrogen purge flow rate of $50 \mathrm{ml} \mathrm{min}^{-1}$

\subsection{Resistance Measurement}

A high resistance meter (HP-4329A, Agilent, CA) with a two-point probe method was used. Resistivity measurements were performed on TPU/G (with different wt $\%$ of $\mathrm{G}$ ). The specimen was prepared by cutting a small part from the TPU/G round compact disc and the surface of the specimen was cleaned by rubbing with distilled water according to ISO $3915 ; 1981$, then allowing it to dry in air at room temperature for three hours. After cleaning, TPU/G specimens were coated by silver coating to ensure good electrical contact. The distance between the probes was larger than the radius of the contact area between the probes and the sample surface. The value of resistance was recorded after a period of 60 seconds, which is known as the time of electrification, to leave measurement to settle. The resistivity (R) was calculated through the slope of I-V curves, with Ohm's law.

\section{Results and Discussion}

TPU and graphene were mixed by dry (mechanical) mixing technique, then the mixture was hot compacted as illustrated in Fig. 1. The solvent (wet) mixing technique is illustrated in the supporting Fig. S1. The dimensions of the prepared compact disc of TPU/G nanocomposite were selected according to ASTM standards for the compression molding technique. The main purpose of producing the round compact discs of TPU/G to be used directly as compression test specimens as shown in Fig S2 and to avoid sharp edges. As a result, this reduces stress concentrations and improves the densification along the sample[21]. It was intriguing to compare the interface between $\mathrm{G}$ nanoparticles and TPU particles size in the two mixing techniques. Dry mixing technique entailed excellent properties due to high rotational speed of the container allowing homogeneity of the TPU/G mixture. Moreover it is more hygienic, dust-free, time-saving and cost effective. While on the other hand, $G$ nanoparticles in solvent mixing inclined to agglomerate and inhomogeneous dispersion occurred during evaporation of solvent. This indicates that polymer degradation has occurred due to solvent effect. Long time exposure to stirring induces defects in graphene sheets which are detrimental to the nanocomposite properties. Agglomeration occurs during slow solvent evaporation with high content of $\mathrm{G}$, resulting in inhomogeneous distribution of G in TPU matrix[13].

\section{Dry mixing}
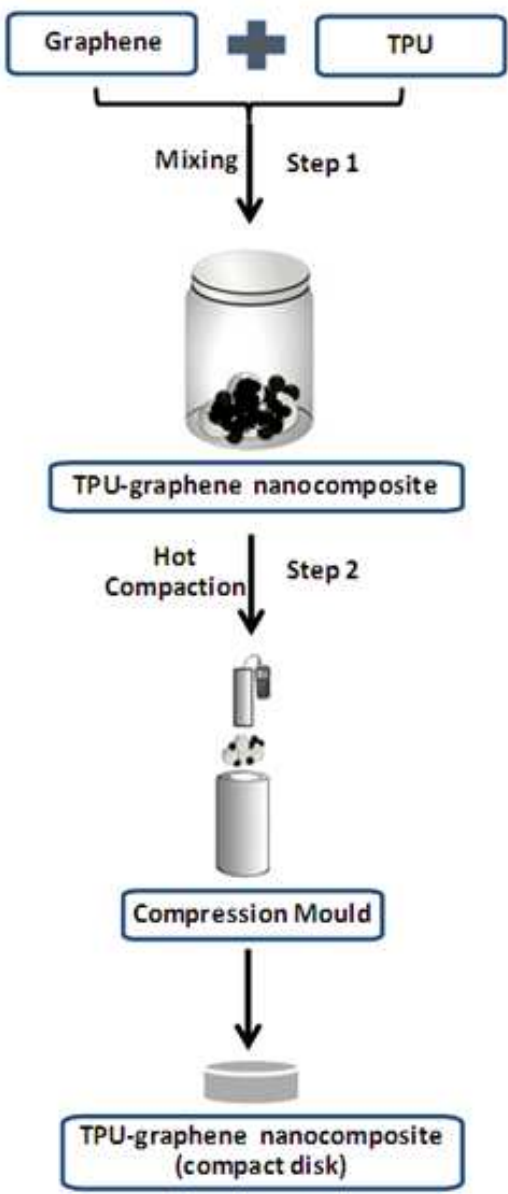

Figure 1. A Schematic illustration of the dry (mechanical) mixing process of TPU/G nanocomposite followed by compression molding.

The mechanical testing performed on TPU/G nanocomposite samples showed promising compressive strength results. Other characterization techniques including MFI, TGA, and resistivity measurement were performed on the mechanically mixed samples. In order to assess the flow of the polymer, MFI test was performed to indicate the relation between wt. $\% \mathrm{G}$ and its influences on the viscosity of the TPU/G nanocomposite. The increase of the viscosity can also decrease the dispersion of the $G$ particles inside the TPU matrix. Moreover, TGA tests approved that $\mathrm{G}$ enhanced the thermal stability of the TPU. On the other hand, the electrical resistivity was evaluated by measuring the conductivity to assess the electrical properties with different wt. \% G. The morphology of the mechanical mixed samples was investigated by SEM to gain more insights into the effect of increasing wt \% G on the mechanical behavior of the produced TPU/G nanocomposites, and investigate the distribution of $\mathrm{G}$ within the TPU matrix. The particle size distribution was determined by means of Image-J software to validate the findings of the SEM examinations. 


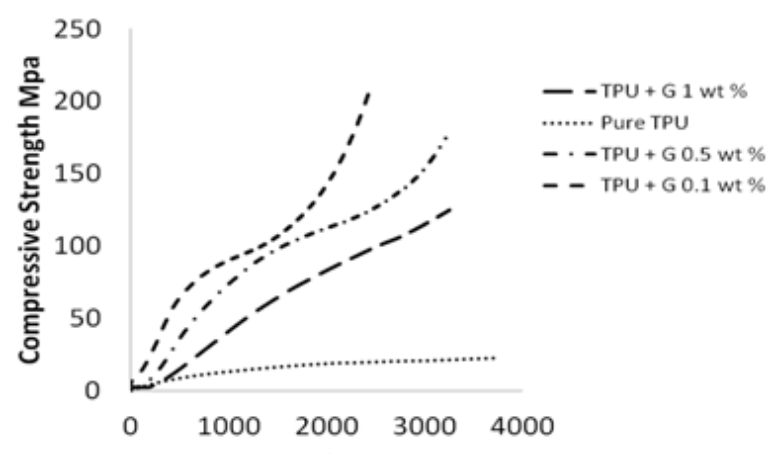

Figure 2. Compressive strength of Pure TPU and different wt \% of G. (e.g. $0.1,0.5,1 \mathrm{wt} . \%$ ) in TPU/G Nano composite prepared by dry (mechanical) mixing

The compressive strength resulting from reinforcement of $\mathrm{G}$ into the TPU was indicated by the plot in Fig. 2. The compressive strength of 0.1 wt. \% G reached $200 \mathrm{MPa}$ which shows a substantial increase in compressive strength compared to pure TPU specimen of $25 \mathrm{MPa}$. Also, adding 0.5 wt. $\%$ G. increases the compressive strength but only to $170 \mathrm{MPa}$. Moreover, The compressive strength increases by adding $1 \mathrm{wt}$. $\%$ of $\mathrm{G}$ to $130 \mathrm{MPa}$. The minimum improvement achieved was with 1 wt. \% G which is still very promising. There is a trend of increasing the diameter size of $\mathrm{G}$ nanoparticles with the increase of the $\mathrm{G}$ wt \%. The increase in compressive strength with the addition of $G$ with increasing wt. percentages is attributed to the extensive carbon network in its structure. The interfacial bond between $G$ nanoparticles and TPU chains is stronger than pure TPU bonds as $G$ is embedded through the TPU soft and hard chains. It is suggested that $\mathrm{G}$ is mechanically dispersed into the TPU during compression molding process. A recent report shows that shear forces during melt mixing disentangle the $\mathrm{G}$ aggregates and disperse the $\mathrm{G}$ within the TPU matrix[22]. Another possible explanation for the decrease of compressive strength upon increasing wt. $\% \mathrm{G}$ is that the interfacial bond between the phases started to weaken as it separates the TPU chains and G behave as holes or nanostructures flaws which as a result inducing local stress concentrations and reducing the transfer between the TPU matrix and $G[23]$. At 1 wt $\% \mathrm{G}, \mathrm{G}$ nanoparticles start to agglomerate and form cracks thus causing a decrease in the compressive strength[24].

Pure TPU and pure $\mathrm{G}$ have no aggregated features as shown in Fig. 3(A, B) respectively, while the TPU / $0.1 \mathrm{wt} \%$ $G$ has irregular shape, possibly clusters form leading to stronger bonds between TPU and G nanoparticles as shown in Fig. 4A. By increasing the wt. $\%$ of $\mathrm{G}(0.5 \mathrm{wt} \%$ and 1 wt \%) in the mechanically mixed samples, it was expected to increase the compressive strength beyond $200 \mathrm{MPa}$. However, the compressive strength decreased to $130 \mathrm{MPa}$ as mentioned previously. It was found that addition of $1 \mathrm{wt} \% \mathrm{G}$ to the TPU matrix created larger agglomerations compared with the 0.5 wt $\% \mathrm{G}$ as reflected in Fig. $4 \mathrm{~B}$ and $\mathrm{C}$, respectively. The observed decrease in compressive strength could be explained by the lack of $G$ uniform distribution due to clustering, which attributed to the alteration of the function of $\mathrm{G}$ nanoparticles from being strengthening filler to inclusions with less cohesion at the TPU-G interfaces. As a result of which induced cracking associated with premature failure occurred.
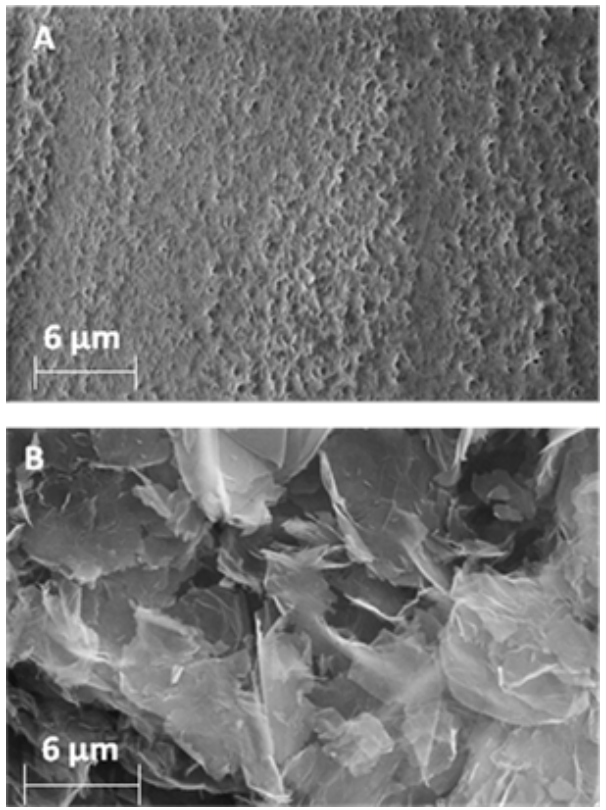

Figure 3. Scanning electron microscope images of (A) Pure TPU, (B) Pure G
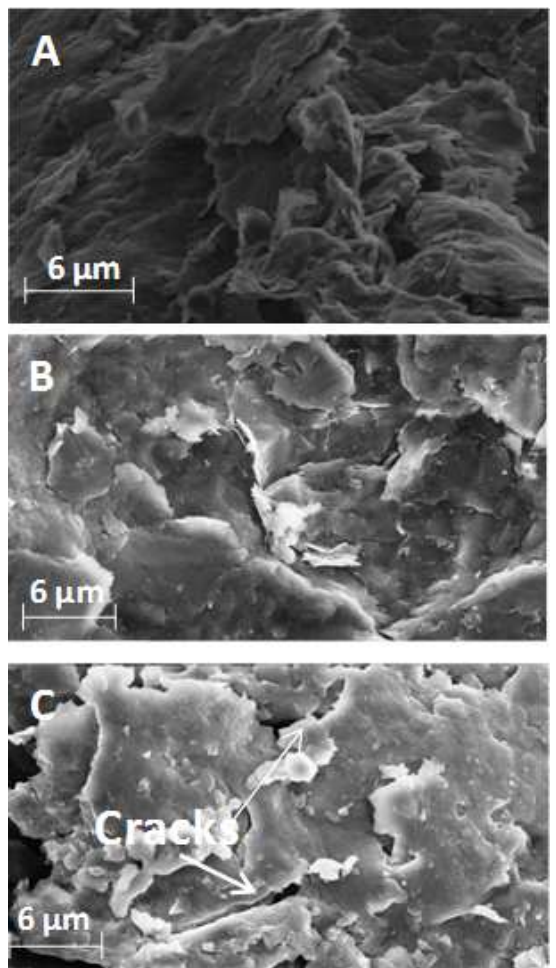

Figure 4. Scanning electron microscope images of TPU/G nanocomposite with different wt \% of $G:$ (A) 0.1 wt. \% G (B) 0.5 wt. \% G(C) 1 wt. \% G

However, the small size distribution at $0.1 \% \mathrm{TPU} / \mathrm{G}$ confirms homogenous dispersions of $\mathrm{G}$ as shown in Fig.5A. The $\mathrm{G}$ particles size measured is $0.105,0.48$ and $1.78 \mu \mathrm{m}$ for 
the $0.1,0.5$ and $1.0 \mathrm{G} \mathrm{wt} \%$, respectively. The increase in the diameter size as the $\mathrm{G}$ wt \% increases is obvious in Fig. 5B and $5 \mathrm{C}$.
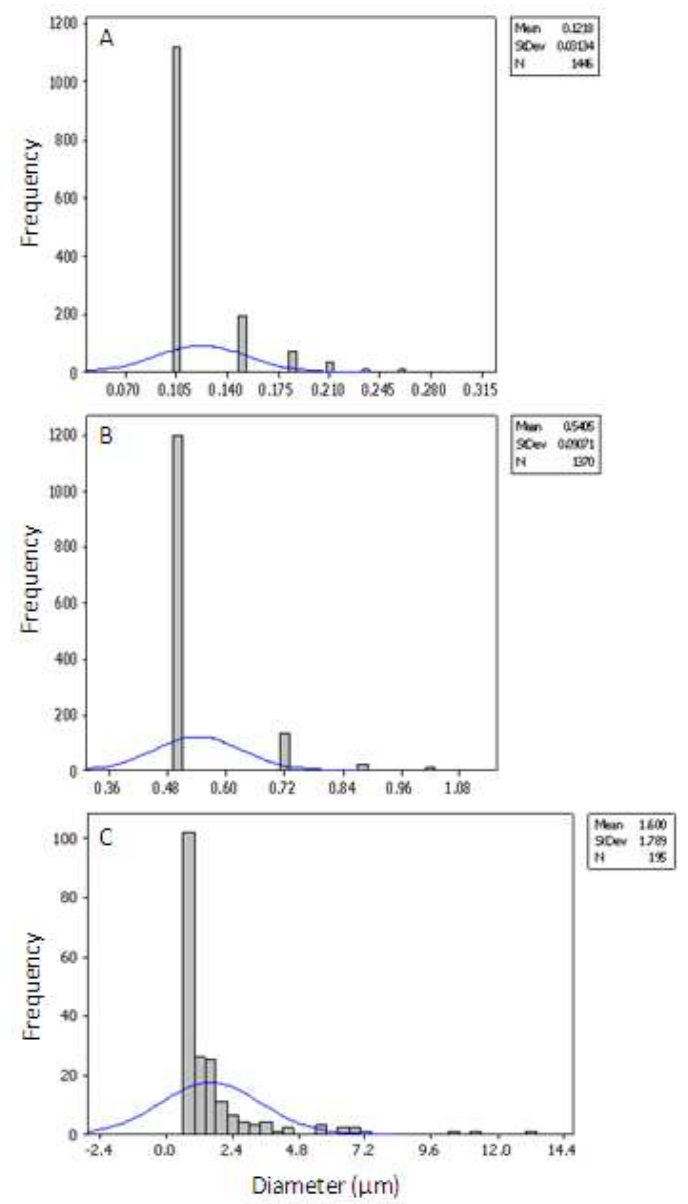

Figure 5. Size distribution of TPU/G nanocomposite with different $w t \%$ of G: (A) $0.1 w t \% G$ (B) $0.5 w t \% G(C) 1 w t \% G$

Moving to the composite physical properties, Not only G plays an important role to improve the mechanical properties but also the $0.1 \mathrm{wt} . \% \mathrm{G}$ reduces the viscosity of TPU melt significantly and makes the processing more easier. It was reflected from the results that the $0.1 \mathrm{wt} \% \mathrm{G}$ shows a higher melt flow index (lower viscosity) than $0.5 \mathrm{wt} \%$ and $1 \mathrm{wt} \%$ G. The increase in G \% decreases the MFI as illustrated in Fig. 6.

The mechanical success for any polymer nanocomposites is governed by the successful transfer of load between the matrix and the reinforcement. Lower melt flow indices (i.e. higher viscosity) can be attributed to higher shear force needed between $\mathrm{G}$ nanoparticles and TPU. It is also related to the high surface area of TPU/ G compared to pure TPU[25]. The transfer of load is better at lower $\mathrm{wt} \%$ of $\mathrm{G}$ (lower viscosity) added to the TPU, thus confirming the previous compressive strength results. The viscosity of all the TPU/G samples increases with increasing $\mathrm{G} w \mathrm{w} \%$. This is most probably due to the formation of agglomerations leading to less dispersion and higher shear force where the two components act as a barrier to one another[26].

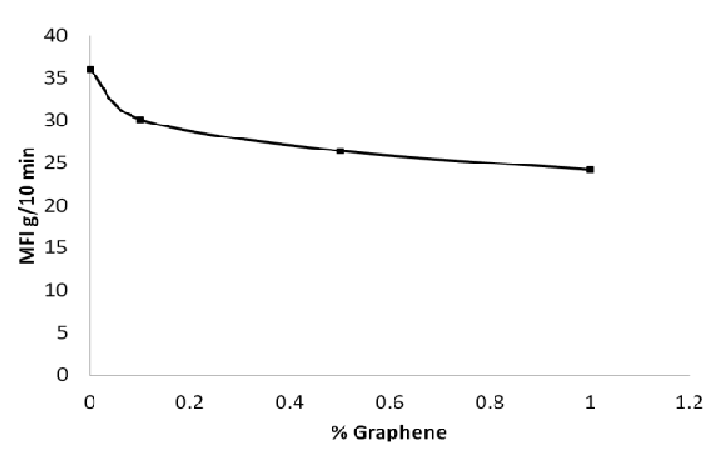

Figure 6. Melt flow index of different wt \% of TPU/ G nanocomposite prepared by dry (mechanical) mixing

It is quite interesting to improve the thermal degradation of the nanocomposite to be used in applications that require higher thermal stability. TGA results showed that there is an increase in temperature with the increase of G-content indicating an enhancement of the thermal stability upon increasing the $\mathrm{G}$ content as shown in Fig. 7. The incorporation of $\mathrm{G}$ in the TPU matrix increased the decomposition temperature. This enhanced the thermal stability of the TPU/G nanocomposite as a result of the interfacial interactions produced between G and TPU. Thermal stability investigations widen the scope of applications of polymer nanocomposites together with the mechanical behavior[27, 28]

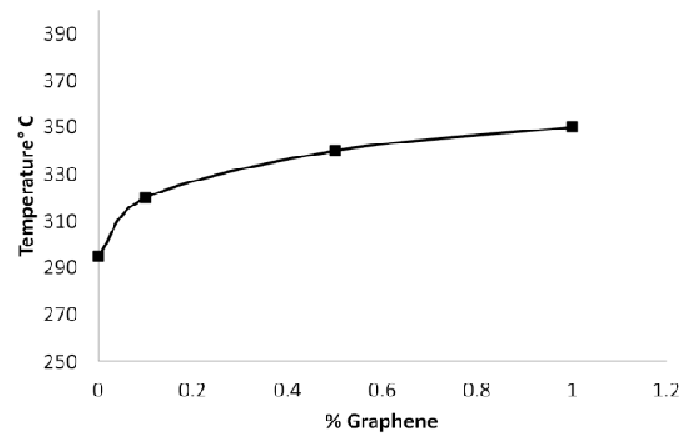

Figure 7. Thermogravimetric analysis of different wt \% of TPU/ G nanocomposite prepared by dry (mechanical) mixing

In addition to enhanced thermal stability, the electrical conductivity of the disc was improved which widens the scope of further applications. It was noticed that electrical resistivity values increased with the increase of $G$ content as illustrated in Fig. 8. There is a significant shift in the increase from the specimen with pure TPU, which is considered as an insulator and the one with the lowest reinforcement. Then there is a surprising increase with the addition of $0.5 \mathrm{wt} \%$ and the $1 \mathrm{wt} \% \mathrm{G}$. Studying the electrical properties of TPU/G showed significant improvement in electrical conductivity thus extending the applications to conductive cables and tubes. The highly dispersed G nanoparticles in the TPU/G nanocomposite significantly enhanced the electronic conductivity and allowed the electrochemical activity of the polymer nanocomposite to be efficiently utilized. G functions as an electron transport 
pathway and enables fast charge transfer, compared to reinforcing with carbon nanotubes, that has an irregular arrangement and defects resulting in lowering the conductivity of the nanocomposite. Increasing $\mathrm{G}$ wt \% further increases the electronic conductivity as shown in Fig. 8. The increase in G-content leads to the formation of a conducting network with a large number of charge carriers traveling though the entire network, and thus higher active material utilization ratio[29].

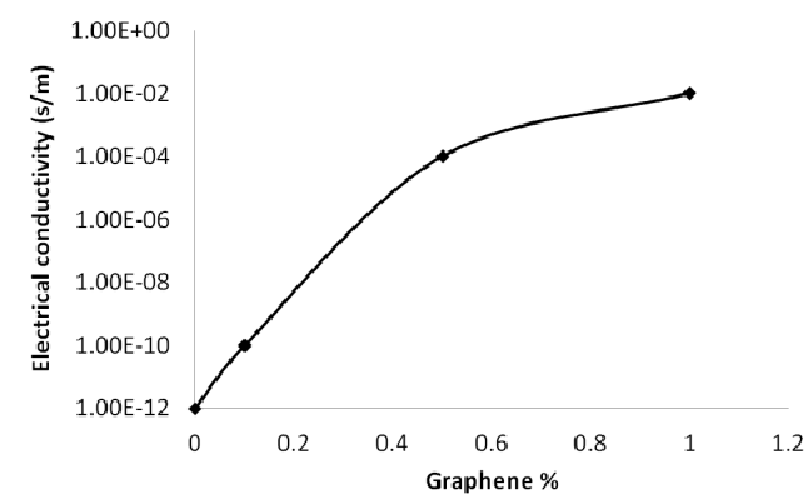

Figure 8. Electrical conductivity of different wt \% of TPU/ G nanocomposite prepared by dry (mechanical) mixing

Table 1 summarizes the results of the different characterization techniques performed on the TPU/G nanocomposite with both mixing techniques. Three specimens from each TPU/G nanocomposite sample were tested and an average value was recorded.

Table 1. Average values of mechanical properties for different $w t \%$ of $G$

\begin{tabular}{|c|c|c|c|c|c|}
\hline \multicolumn{2}{|c|}{$G W t . \%$} & 0 & 0.1 & 0.5 & 1 \\
\hline \multicolumn{2}{|c|}{ Diameter size $(\mu m)$} & 0 & $0.105 \pm 5$ & $0.48 \pm 9$ & $1.72 \pm 7$ \\
\hline \multirow{5}{*}{ Dry Mixed } & $\begin{array}{l}\text { Compressive strength } \\
(M P A)\end{array}$ & $h_{25 \pm 1.6}$ & $200 \pm 3.2$ & $170 \pm 0.5$ & $130 \pm 2.3$ \\
\hline & $M F I(g / 10 \mathrm{~min})$ & $55 \pm 1.3$ & $53.2 \pm 1.4$ & $51.7 \pm 1$ & $50.1 \pm 0.3$ \\
\hline & $T G A\left({ }^{\circ} \mathrm{C}\right)$ & $295 \pm 3$ & $320 \pm 1$ & $340 \pm 2$ & $350 \pm 2$ \\
\hline & $\begin{array}{l}\text { *Electrical resistivity } \\
(\mathrm{s} / \mathrm{m})\end{array}$ & $y_{1.00 E-12}$ & $00 E-$ & $1.00 E$ & $1.00 E-02$ \\
\hline & $\begin{array}{l}\text { Compressive strength } \\
(M P A)\end{array}$ & $h_{25 \pm 1.5}$ & $115 \pm 2.1$ & $100 \pm 0.8$ & $70 \pm 1.9$ \\
\hline \multirow{3}{*}{$\begin{array}{l}\text { Solvent } \\
\text { mixed }\end{array}$} & $M F I(g / 10 \mathrm{~min})$ & $36 \pm 1.5$ & $30.1 \pm 0.9$ & $26.4 \pm 0.6$ & $24.2 \pm 2$ \\
\hline & $T G A\left({ }^{\circ} \mathrm{C}\right)$ & $300 \pm 1$ & $300 \pm 4$ & $320 \pm 3$ & $330 \pm 2$ \\
\hline & $\begin{array}{l}\text { *Electrical resistivity } \\
(\mathrm{s} / \mathrm{m})\end{array}$ & $y 1.00 E-$ & $.00 E-1$ & $1.00 E-$ & $1.00 E-04$ \\
\hline
\end{tabular}

$* \pm 0.3 \mathrm{~V}$

During processing, there were several concerns regarding wet mixing process. Graphene incline to agglomerate and inhomogeneous dispersion can occur during evaporation leading to low surface energy and decreased mechanical properties due to the effect of solvent as shown in Fig. 9. Long time exposure to stirring induces defects in graphene nanoparticles which are detrimental to the composite properties. Agglomeration occurred during slow solvent evaporation, resulting in inhomogeneous distribution of graphene in TPU matrix. When G content increases, interfacial adhesion between the phases becomes weak, and the nanofillers behave as holes or nanostructured flaws, inducing local stress concentrations and reducing the transfer between the matrix and the filler. Besides, wet mixing is not ideal for industry production, owing to the low yield and high production cost.

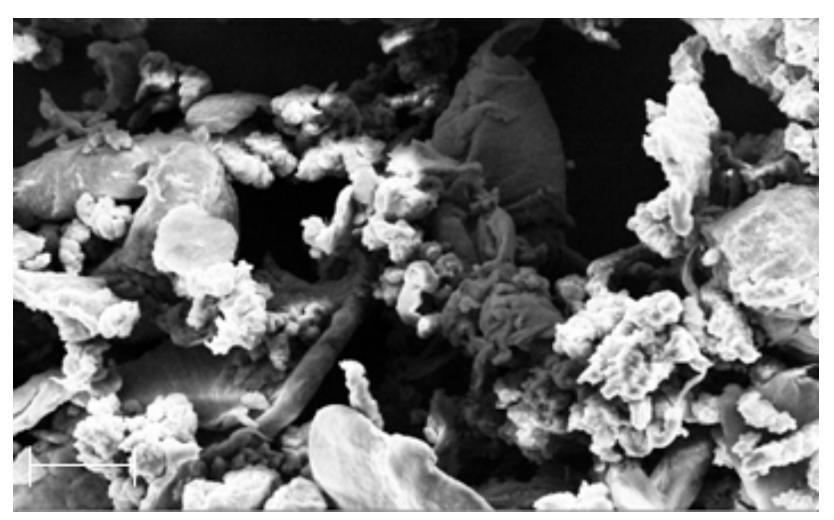

Figure 9. Scanning electron microscope images of TPU/G nanocomposite with 1 wt $\%$ of $G$ (wet mixing)

Thus, there is still a huge demand for fabrication techniques based on dry mixing. The production process based on dry mixing is hygienic and dust-free because the product is mixed in independent containers of variable sizes. There is no contamination, emissions nor extra cleaning. It is a time-saving process due to simple and fast mounting of the container. It is suggested that the surfaces of the particles become activated and disordered during the dry mixing process which improves the mechanical properties as shown in table 1 . The compressive strength at $0.1 \mathrm{wt} \%$ improved by 85 MPA compared to wet mixing. The melt flow index increased significantly by $23 \mathrm{~g} / 10 \mathrm{~min}$. This caused a decrease in the viscosity of the mixture allowing smooth processing. There is an enhancement in the thermal stability by 20 degrees in dry mixing due to an increase in transition temperature by the formation of different crystallization forms. Finally the electrical resistivity enlarged by 1 fold due to increasing the distance in which an electron can travel freely without having its path disrupted by scattering[30].

\section{Conclusion}

TPU/G nanocomposites were prepared using two processing techniques: mechanical mixing followed by compression moulding and wet mixing. Dry mixing results showed an overall improvement in the mechanical properties compared with wet method, due to the excellent homogeneity of the mixture due to interaction of rotation. A surprising shift in compressive strength was produced at 0.1 wt \% G. The strength increased seven folds compared to pure TPU. The lowest viscosity (highest MFI) was obtained at $0.1 \mathrm{wt} \% \mathrm{G}$ allowing easier mixing and processing of the TPU/G nanocomposite. Moreover, there was a significant increase and shift in the temperature occurred at the lowest 
reinforcement content $(0.1$ wt $\% \mathrm{G})$ with a slow but continuous increase with increasing G-content. Electrical conductivity was enhanced by adding $0.1 \mathrm{wt} \% \mathrm{G}$ and increased by adding $0.5,1 \mathrm{wt} \% \mathrm{G}$. The choice of the suitable wt. $\% \mathrm{G}$ depends on the application, if compressive is sacrificed for electrical conductivity and thermal stability then $0.5,1 \mathrm{wt}$. \% $\mathrm{G}$ will be suitable. Potential applications for $\mathrm{G}$ as a nanofiller has opened a new dimension for the production of light weight, low cost solar cells, packaging films, automobile components and biomedical application including medical implants

\section{Appendix}

TPU/G nanocomposites were prepared by wet (solvent) mixing process, where TPU pellets were dissolved in Tetrahydrofouran (Sigma Aldrich, 99\% purity), with constant stirring to form a clear homogeneous liquid phase. Graphene nanofiller powder was then added to the TPU solution with continued stirring for $60 \mathrm{~min}$. The resulting TPU/G nanocomposite was left to dry in air at room temperature till constant weight. The whole process is shown in Fig. S1. The desired shape of the TPU/G samples produced was compact disc as illustrated in Fig. S2 with the specified dimensions for Pure TPU: Fig S2 (A,B), and TPU/G nanocomposite Fig. $\mathrm{S} 2(\mathrm{C}, \mathrm{D})$, respectively, according to ASTM of the compression molding technique.

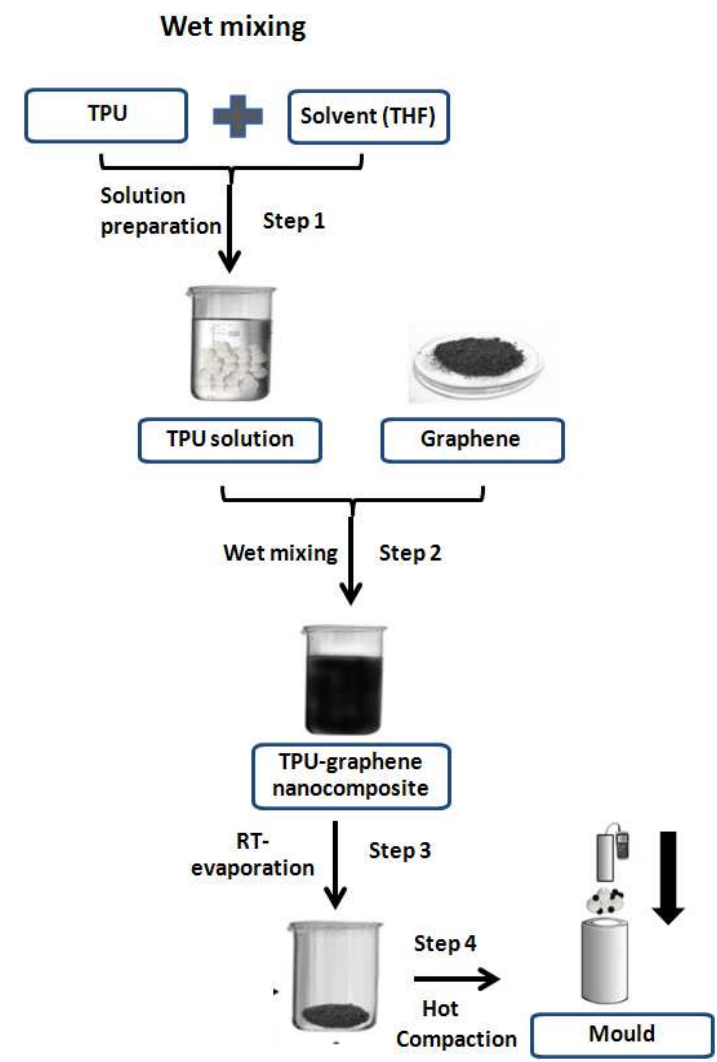

Figure S1. A Schematic illustration of the solvent (wet) mixing process of TPU/G nanocomposite followed by compression molding.
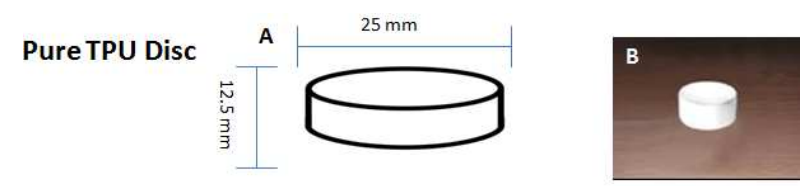

TPU /G Disc
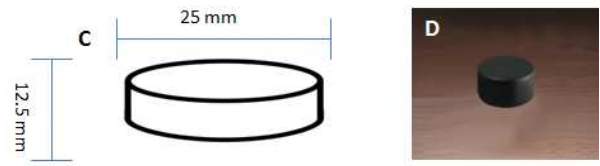

Figure S2. Compact discs of pure TPU (A) and TPU/G nanocomposite (C).Prepared discs with different colors: white (B) for pure TPU and black (D) for TPU/G nanocomposite.

The increase in compressive strength with the addition of graphene with different percentages is attributed to the extensive carbon network in its structure. The interfacial bond between $\mathrm{G}$ nanoparticles and TPU chains is stronger than Pure TPU bonds as G is embedded through the TPU soft and hard chains as shown in Fig. S3.

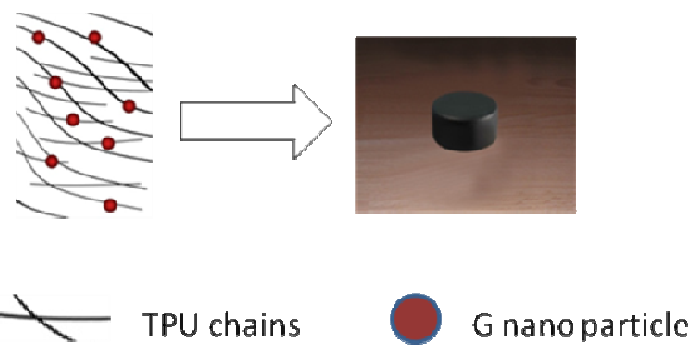

Figure S3. Schematic illustration of dispersion of $G$ nanoparticles inside TPU matrix

A possible explanation for the decrease in compressive strength (140 MPa) fabricated by solvent mixing as shown in Fig. S4 in comparison with the compressive strength (200MPa) of the dry mixing technique is that $\mathrm{G}$ nanoparticle incline to agglomerate and inhomogeneous dispersion can occur during evaporation leading due to low surface energy and decreased mechanical properties caused by the effect of solvent. However this can be decreased by placing TPU/G suspension on a rotating substrate (spin casting) or dropping it on a hot substrate (drop casting).

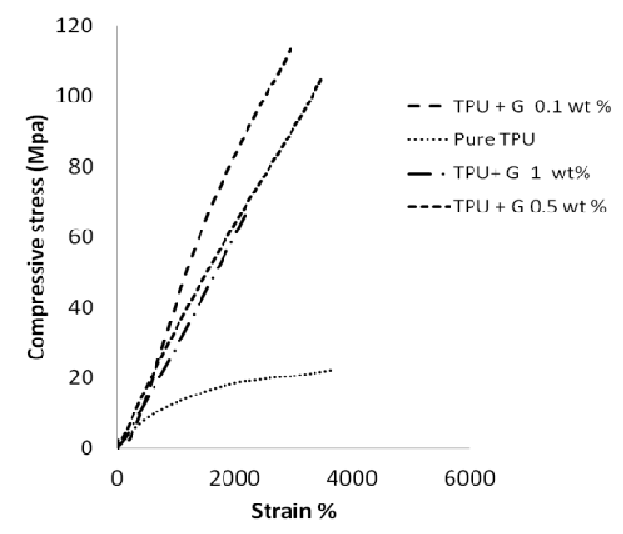

Figure S4. Compressive strength of Pure TPU and different wt. \% of G. (e.g. $0.1,0.5,1 \mathrm{wt} . \%)$ in TPU/G Nano composite prepared by solvent (wet) mixing 
Long time exposure to stirring induces defects in graphene sheets which are detrimental to the nanocomposite properties. Agglomeration occurs during slow solvent evaporation with high content of $\mathrm{G}$, resulting in inhomogeneous distribution of $G$ in TPU matrix. The results clearly show that the mechanical properties are lower in value for the solvent-mixed sample relative to the dry-mixed sample. This indicates that polymer degradation has occurred, a concern highlighted by other researchers and observed in this study[16].

\section{References}

[1] J. L. Vickery, A. J. Patil, S. Mann, "Fabrication of graphene-polymer nanocomposites with higher-order three-dimensional architectures" Adv. Mater., Vol 21, 2180-2184, 2009.

[2] H. Kim, A. A. Abdala, and C. W. Macosko, "Graphene/Polymer Nanocomposites" Macromolecules, Vol43, pp 6515-6530, 2010

[3] C. N. R. Rao, A. K. Sood, R. Voggu, and K. S. Subrahmanyam. "Some Novel Attributes of Graphene" J. Phys. Chem. Lett. ,Vol 1, pp 572-580, 2010.

[4] K. B. Carey, T. Jerome, and S. Karna, "Carbon Nanotube Aluminum Matrix Nanocomposites" Weapons and Materials Research Directorate, Army Research Laboratory, Vol 1, 2010 .

[5] M. J. Allen, V. C. Tung, and R. B. Kaner, "Honeycomb Carbon: A Review of Graphene" Chem. Rev. Vol 110, pp $132-145,2010$

[6] K. T. Lau, "Recent Research in Nanotube/Nanoclay Related Polymer Composites" Twelve International Conference on Composites/Nano engineering, ICCE-12, Tenerife, Spain, August 2005

[7] N.A. Ochekpe, O. Patrick, and C.N. Wuluka,'Nanotechnology and Drug Delivery. Part 1: Background and Applications" Tropical Journal of Pharmaceutical Research, pp.265-274, 2009.

[8] D. Xiao, X. Zhao, Y. Feng, and P. Xiang, W. Zhang, 'The structure and dynamic properties of thermoplastic polyurethane elastomer/ hindered phenol hybrids" Journal of Applied Polymer Science ,pp. 2143-2150,2010

[9] A.V. Pinheiro, D. Han, W.M. Shih, and H.Yan, 'Challenges and opportunities for structural DNA nanotechnology" Nature Nanotechnology, Vol.6, pp. 763-772, 2011.

[10] J. Bergstrom, and M.C. Boyce, "Mechanical behavior of particle filled elastomers." Mech. Phys. Solids, in press.

[11] M. Aurilia, F. Piscitelli, L. Sorrentino, and M. Lavorgna, ' Detailed analysis of dynamic mechanical properties of TPU nanocomposite: The role of the interfaces". European Polymer Journal, Vol. 47, pp. 925 -936, 2011.

[12] Y. Wang, Z. Shi, J. Fang, H. Xu, and J. Yin, "Graphene oxide/polybenzimidazole composites fabricated by a solvent-exchange method" Carbon, Vol. 49, pp.1199-1207, 2011.
[13] A.M. Esawi, H.G. Salem, H.Hussein, and A. Ramadan, "Effect of Processing Technique on the Dispersion of Carbon Nanotubes Within Polypropylene Carbon Nanotube-Nanocomposites and Its Effect on Their Mechanical Properties" Polymer Composites, Vol. 31, pp.772, 2009.

[14] E.W. Gacitua, A.B. Ballerini and J. Zhang, "Polymer nanocomposites: synthetic and natural fillers a review" Maderas.Ciencia y tecnologia,Vol. 13, pp.159-178,2005.

[15] Y. Jichun, Q.H. Bing, L. Zonghoon, A. Byungmin, R.Steve, and J. M. Schoenun, "A tri-modal aluminum based composite with super-high strength." Scripta Materialia,Vol. 53, pp.481-486, 2005.

[16] E. Ionescu, A.Francis, R. J. Riedel, "Dispersion assessment and studies on AC percolative conductivity in polymer-derived $\mathrm{Si}-\mathrm{C}-\mathrm{N} / \mathrm{CNT}$ ceramic nanocomposites" Material Science, Vol. 44, pp. 2055-2062, 2009

[17] H. Quan, B. Zhang, Q. Zhao, K. Richard, K. Yuen, and K. Y. Robert,"Facile preparation and thermal degradation studies of graphite nanoplatelets(GNPs) filled thermoplastic polyurethane (TPU) nanonanocomposites" Nanocomposites; Part A,Vol. 40,pp. 1506-1511,2004.

[18] A. K Barick, and D.K.Tripathy, ” Effect of nanofiber on material properties of vapor-grown carbon nanofiber reinforced thermoplastic polyurethane (TPU/CNF) nanocomposites prepared by melt compounding" Nanocomposites; Part A,Vol. 41, pp.1471-1482,2010

[19] T.M. Madkour, T. M and F.M. Hagag, W. Mamdouh, and R.A Azzam, "A Nanoscale Molecular-level modeling and experimental investigation into the high performance nature and low hysteresis of thermoplastic polyurethane/multi-walled carbon nanotubes nanocomposites Polymer,Vol. 53, pp.5788-5797,2012

[20] Z. Spitalsky, D.Tasis, K. Papagelis, and C.J. Galiotis Carbon nanotube-polymer composites: Chemistry, processing, mechanical and electrical properties". .Poly. Sci, Vol. 35, pp.357-401, 2010.

[21] D6641 ASTM standard test method for compressive properties of polymer matrix composites; Pennsylvania; ASTM international.

[22] E. Thostenson, "Advances in the Science and Technology of Carbon Nanotubes and their Nanocomposites: A Review." Nanocomposites Science and Technology, Vol. 61, pp.1899-1912, 2001.

[23] N. Jing, Q. Xue, C.Ling, M. Shan, T. Zhang, X. Zhou, and Jiao, "Effect of defects on Young's modulus of graphene sheets: a molecular dynamics simulation 'RSC $A d v$., Vol.2, pp.9124-9129, 2012.

[24] J. M. Cowie ,and V.Arrighi, Polymers: Chemistry and Physics of modern materials. 3 rd ed., CRC Press, 2008 ..

[25] B. Lin, U. Sundararaj, and P.Guegan, ” Effect of mixing protocol on compatibilized polymer blend morphology" Polymer Engineering and Science, Vol. 46,pp. 691-702,2006.

[26] A. Funck, "Polypropylene Carbon Nanotube Nanocomposites by in Situ Polymerization." Nanocomposites Science and Technology, Vol. 67, pp.906-915, 2007. 
[27] A.L. 2. College of Science, China University of Petroleum, Qingdao, P. R. ChiValentini, "Morphological Characterization of Single-Walled Carbon Nanotubes-Nanocomposites." Nanocomposites Science and Technology, Vol. 63, pp 1149-1153, 2003.

[28] S. Archana, S. Patole, H. Kang, J. Yoo, T. Kim, J. Ahn, Col, "A facile approach to the fabrication of graphene/polystyrene nanocomposite by in situ microemulsion polymerization"
Inter. Sci. ,Vol. 350,pp.530-533, 2010

[29] C. Nan, Y. Shen, and J.Ma, "Physical Properties of Nanocomposites near Percolation” Annu. Rev. Mater. Res., Vol. 40, pp.131-151, 2010.

[30] Li, Q., Dunn, E.T., Grandmaison, E. W. and Goosen, M.F.A. "Application and Properties of Chitosan" J. Bioact. Compat. Polym., Vol7,pp. 370-397,1992 